\title{
Perfiles asociados al consumo de alcohol en adolescentes colombianos
}

\author{
Augusto Pérez-Gómez ${ }^{1,2^{*}}$, Carlos Lanziano ${ }^{1}$, María Fernanda Reyes-Rodríguez ${ }^{1,2}$, \\ Juliana Mejía-Trujillo ${ }^{1,}$ y Francisco Cardozo-Macías ${ }^{1}$ \\ ${ }^{1}$ Corporación Nuevos Rumbos, Bogotá, Colombia, ${ }^{2}$ Universidad El Bosque, Bogotá, Colombia
}

Recibido, julio 4/2017

Concepto de evaluación, noviembre 17/2017

Aceptado, enero 31/2018
Referencia: Pérez-Gómez, A., Lanziano, C., ReyesRodríguez, M.F., Mejía-Trujillo, J. \& Cardozo-Macías, F. (2018). Perfiles Asociados al Consumo de Alcohol en Adolescentes Colombianos. Acta colombiana de Psicología, 21(2), 258-269. doi: http://www.dx.doi. org/10.14718/ACP.2018.21.2.12

Resumen

El consumo de alcohol en adolescentes tiene una serie de consecuencias negativas desde el punto de vista de salud, comportamiento y riesgo de consumo problemático a edades tempranas. En este estudio, se aplicó un instrumento de 24 preguntas a 9348 estudiantes de ambos sexos en nueve ciudades de Colombia, de los cuales 7625 fueron incluidos en este análisis por haber consumido alcohol. Específicamente, se exploraron temas como la edad de inicio de consumo, el tipo de alcohol y las cantidades ingeridas, así como los sitios y la facilidad de adquisición, la presencia de adultos, el consumo en el colegio y las actitudes frente a las restricciones legales, entre otros. Se hizo un análisis de correspondencias múltiples que determinó inicialmente tres factores que fueron después clasificados en dos grupos y, por último, en cuatro subgrupos en función de cuatro categorías de consumo: experimental, selectivo, regular y alto, que representan dos grandes grupos: bajo y alto consumo. De este análisis surgieron perfiles bastante claros que muestran diferencias importantes, especialmente entre dos grupos: los de bajo consumo, que son los más jóvenes, no toman solos, no han estado en fiestas con alcohol, nunca toman en el colegio ni durante varios días seguidos; y los de alto consumo, que son un poco mayores, cuando toman se emborrachan, tienden a tomar todas las semanas, han tomado en el colegio y antes de entrar por las mañanas, se han metido en problemas por tomar y rechazan las restricciones a los menores de edad o que se sancione a quienes les venden.

Palabras clave: adolescentes, alcohol, prevalencias, perfiles, correspondencias múltiples.

\section{Profiles associated with alcohol consumption in Colombian adolescents}

\begin{abstract}
Alcohol abuse in adolescents has several negative consequences on health, behavior and risk of becoming involved in problematic consumption at early ages. In this study a 24-item questionnaire was answered by 9,348 school adolescents of both sexes in nine Colombian cities. 7,625 students were included in this analysis due to their consumption of alcohol. The questions inquired about age of first intake, kind and amounts of alcohol consumed on each occasion, places and availability, presence of adults, alcohol use at school and attitudes toward legal restrictions, among others. A multiple correspondence analysis was carried out, generating four factors which were condensed later into two groups, and finally in four sub-groups based on categories of alcohol use: experimental, selective, regular and high, representing two categories: low and high use. The analysis produced identifiable profiles, quite different especially for two categories: the 'low consumption' group, that is younger, never drinks alone, does not attend parties where alcohol is offered to minors, has never drunk at school or during several days. In the 'high consumption' group are those over 16 years of age, who generally get drunk when they drink, have taken alcohol while at school and before arriving to school, have been in trouble because of alcohol and consider as inappropriate the legal restrictions to minors and the sanctions imposed to adults who sell or offer alcohol to minors.

Key words: adolescents, alcohol, prevalences, profiles, multiple correspondences
\end{abstract}

* Calle 108 A No 4-15, Bogotá, Colombia. Tel: +571 6191152, aperez@nuevosrumbos.org En el presente artículo algunos apartados del marco teórico hacen parte de una obra que ha sido objeto de divulgación previa en el informe final de Pérez-Gómez, A., Mejía-Trujillo, J., Reyes-Rodríguez, M. F., \& Cardozo-Macías, F. (2015) Consumo de alcohol en menores de 18 años en Colombia: 2015. Bogotá, Colombia: Corporación Nuevos Rumbos. Disponible en: http://nuevosrumbos.org/wpcontent/ uploads/2015/12/Consumo-de-Alcohol-en-menores-de-edad-en-Colombia-2015.pdf 


\title{
Perfis associados ao consumo de álcool em adolescentes colombianos
}

\begin{abstract}
Resumo
O consumo de álcool em adolescentes tem uma série de consequências negativas desde o ponto de vista da saúde, do comportamento e do risco de consumo problemático em idades precoces. Neste estudo, aplicou-se um instrumento de 24 perguntas e 9348 estudantes de ambos os sexos em nove cidades da Colômbia, dos quais 7625 foram incluídos nesta análise por terem consumido álcool. Especificamente, foram explorados temas como a idade inicial de consumo, o tipo de álcool e as quantidades ingeridas, assim como os lugares e a facilidade de aquisição, a presença de adultos, o consumo no colégio e as atitudes frente às restrições legais, entre outros. Foi realizada uma análise de correspondências múltiplas que determinou inicialmente três fatores que depois foram desclassificados em dois grupos e, por último, em quatro subgrupos em função de quatro categorias de consumo: experimental, seletivo, regular e alto, que representam dois grandes grupos: baixo e alto consumo. A partir dessa análise, surgiram perfis bastante claros que mostram diferenças importantes, especialmente entre dois grupos: os de baixo consumo, que são os mais jovens, não bebem sozinhos, não estiveram em festas com bebidas alcoólicas, nunca bebem no colégio nem durante vários dias consecutivos; e os de alto consumo, que são um pouco mais velhos, embriagam-se quando bebem, tendem a beber semanalmente, já beberam no colégio e antes de ir para a aula de manhã, já se meteram em problemas por beber e se opõem às restrições aos menores de idade ou às sanções àqueles que vendem para menores.

Palavras-chave: adolescentes, álcool, correspondências múltiplas, perfis, prevalências.
\end{abstract}

\section{INTRODUCCIÓN}

Diferentes estudios (Ministerio de Justicia y el Derecho, et al. 2011; Ministerio de Justicia y el Derecho, Ministerio de Salud y Protección Social \& Observatorio de Drogas de Colombia, 2014; Pérez-Gómez \& Scoppetta, 2009) muestran que el consumo de alcohol en adolescentes es un problema real en la cultura colombiana. El primer estudio sobre consumo de alcohol en menores escolarizados realizado en siete capitales de departamento y dos municipios pequeños se llevó a cabo en el año 2008 (Pérez-Gómez \& Scoppetta, 2009) y sus principales resultados mostraron índices muy elevados de consumo de alcohol, así como la presencia de un gran número de conductas de riesgo asociadas al beber, como ingerir licor en el colegio o escuela - $\mathrm{O}$ antes de llegar a ella-, beber varios días seguidos, y tener problemas con la policía, en la casa o con personas cercanas por ese motivo. Este primer estudio también reveló un comportamiento indiferente o abiertamente irresponsable de los adultos, quienes proporcionaban el alcohol, lo vendían o toleraban su uso.

El segundo estudio de consumo de alcohol en menores, terminado en diciembre de 2015 (Pérez-Gómez, MejíaTrujillo, Reyes-Rodríguez \& Cardozo-Macías, 2015), buscaba examinar si en esos siete años habían ocurrido cambios, y de qué tipo. La comparación fue posible y pertinente porque se utilizó una metodología idéntica en los dos estudios y se recogieron datos en las mismas zonas geográficas.
Consumo de alcohol en la adolescencia

Las continuas presiones del medio, junto con las crisis "psicológicas" propias de la edad, hacen del adolescente un ser expuesto y vulnerable al consumo de alcohol. Las razones asociadas al consumo son muy variadas, pero hace ya varios años que se le da una importancia particular al disfrute. Fry (2011), por ejemplo, afirma que los jóvenes consumen alcohol con el objetivo de obtener placer en un mundo en el que se considera el hedonismo como fin supremo y en donde la publicidad vende el consumo de licor asociándolo a estados de bienestar, alegría y gozo. En Colombia, como en muchos otros países occidentales, el disfrute del ocio y de las relaciones sociales se encuentra fuertemente asociado al consumo de alcohol y ocurre por lo general los fines de semana en contextos de recreación y esparcimiento. De igual forma, se ha encontrado que en países europeos los jóvenes consumen un promedio de tres tragos el jueves por la noche, cuatro el viernes y cinco o seis el sábado en horario nocturno (Kuntsche \& Labhart, 2012); mientras que, en Colombia, los jóvenes generalmente toman los viernes y los sábados, y el $31 \%$ toma más de dos tragos, el $24 \%$ toma máximo cuatro tragos y el $9 \%$ se embriaga (Pérez-Gómez \& Scoppetta, 2009).

Los menores de edad tienen razones muy variadas para iniciar el consumo: además de la disponibilidad de la sustancia, está la generalización y normalización como una característica propia de la cultura colombiana, las actitudes favorables hacia el consumo, la baja percepción de riesgo, las influencias normativas inadecuadas y las representaciones sociales o creencias positivas frente al consumo de alcohol 
(Becoña, 1999; Fagan, Hanson, Briney \& Hawkins, 2012; Martínez, 2006). Y es importante tener en cuenta que, tal como se ha demostrado en numerosos países, quienes comienzan a consumir alcohol antes de los 14 años de edad tienen hasta 10 veces más probabilidades de tener problemas con el alcohol en la adultez o de consumir otras sustancias que las personas que empiezan a tomar después de los 18 años (Pérez-Gómez, Scoppetta \& Flórez-Alarcón, 2011).

En todos los países del continente americano la edad legal de consumo de alcohol es de 18 años, con excepción de los Estados Unidos, en donde (en la mayor parte de los Estados) es de 21 años. Existe evidencia científica reciente que afirma que el consumo de alcohol en la adolescencia puede tener consecuencias graves sobre diferentes sistemas, sobre todo en el sistema nervioso, tal como se muestra a continuación.

Una de las mayores consecuencias del consumo de alcohol en la adolescencia es el compromiso de varias áreas y funciones del cerebro, en donde el desempeño a nivel intelectual deja de ser el adecuado (Jacobus \& Tapert, 2013). Numerosos estudios (Fein et al., 2013; Jacobus \& Tapert, 2013; Pascual, Pla, Miñarro \& Guerri, 2014; Ward, Lallemand \& De Witte, 2014) han demostrado la afectación, en cierto grado, del aprendizaje a través de refuerzo, el control motor del cuerpo, la formación de la corteza cerebral encargada del procesamiento y almacenamiento de la información -que interviene en la toma de decisiones, la planeación, el raciocinio, la solución de problemas y control de impulsos-, la memoria a largo plazo, la efectividad en las conexiones con los lóbulos parietales, temporales, regiones límbicas, entre otras, y la comunicación entre ambos hemisferios cerebrales; todo esto como consecuencia de la disminución de tálamo, el putamen y la densidad de la materia gris (Fein et al., 2013; Jacobus \& Tapert, 2013), así como de la corteza prefrontal, el hipocampo y la capacidad de difusión del cuerpo calloso (De Bellis et al., 2005).

Asimismo, se ha encontrado que el abuso intermitente de etanol puede producir fenómenos inflamatorios en el hipocampo y en el córtex prefrontal, especialmente en la etapa de neurogénesis activa de la adolescencia, que pueden resultar déficits cognoscitivos (Pascual et al., 2014; Ward et al., 2014), por lo que se puede decir que, teniendo en cuenta que el cerebro solo alcanza su máximo desarrollo hasta los 21 años de edad, el beber alcohol antes de esa edad afecta el progreso madurativo del cerebro. Al respecto, los estudios de la Universidad de Duke (White \& Swartzwelder, 2006) y de la Universidad de San Diego (Brown y Tapert, 2008) muestran de forma clara que en los adultos jóvenes que fueron consumidores frecuentes de alcohol en su adolescencia hay un desempeño pobre en pruebas de memoria verbal y no verbal, así como dificultades para focalizar la atención y en el manejo de abstracciones espaciales -como en la lectura de mapas-.

Además del cerebro, otros órganos y sistemas se ven altamente comprometidos con la ingesta crónica de alcohol; hay por lo menos 25 enfermedades directamente atribuibles al consumo excesivo de alcohol, así como muchas otras que tienen una relación indirecta con dicho consumo o agravan cualquier condición física preexistente (Shield, Parry \& Rehm, 2014). Por ejemplo, con respecto al sistema autoinmune, el alcohol aparentemente predispone o incrementa la severidad y aparición de un gran número de infecciones -debido a la dificultad que tienen las células blancas de combatir bacterias- (Barr, Helms, Grant \& Messaoudi, 2015; Szabo \& Mandrekar, 2009); asimismo, la probabilidad de padecer de diabetes se incrementa considerablemente en jóvenes adultos que consumieron grandes cantidades de alcohol en su adolescencia (Liang \& Chikritzhs, 2014).

Por otra parte, los sistemas cardiovascular y digestivo también se ven altamente implicados en este proceso, ya que la ingestión crónica de alcohol, además de subir la presión sanguínea, conlleva a alteraciones en el ritmo cardíaco y problemas de dilatación y pérdida de la fuerza de contracción del corazón (Vicente-Herrero et al., 2015). Mientras que el hígado -hace más de dos siglos que se sabe-, el encargado del procesamiento metabólico del alcohol, puede padecer graves dolencias asociadas al abuso del mismo. También se ha encontrado que el consumo excesivo de esta sustancia disminuye la capacidad de absorción de nutrientes en el intestino delgado, lo que puede causar problemas de salud en los huesos y en el sistema endocrino (Parés \& Caballería, 2006).

Las repercusiones del consumo de alcohol también pueden encontrarse en afectaciones de la salud mental en los adolescentes. Por ejemplo, se ha observado que los jóvenes que consumen alcohol tienen cuatro veces más probabilidades de sufrir síntomas de depresión que quienes no lo hacen (Briones \& Woods, 2013; Pilatti et al., 2014); y, a su vez, los síntomas depresivos pueden exacerbar o desencadenar otros comportamientos problemáticos, tales como conductas oposicionistas y desafiantes, agresividad, dificultades de aprendizaje y consumo de otras sustancias psicoactivas. De igual forma, el consumo de alcohol hace que el aprendizaje se vuelva mucho más lento en comparación con jóvenes de la misma edad que no consumen, ya que se ven afectados los procesos atencionales, de memoria y de pensamiento (Briones \& Woods, 2013; Ward et al., 2014).

La relación entre alcohol y las conductas violentas ha sido igualmente reconocida en la literatura sobre el tema (Pérez-Trujillo, Reyes, Cabrera y Pérez-Gómez, 2016; Schofield \& Denson, 2013), donde se han reportado 
comportamientos como las riñas (Pridemore \& Grubesic, 2012), los crímenes de acoso, el crimen violento y el daño criminal (Bromley \& Nelson, 2002). Si bien esta relación entre crímenes y consumo de alcohol es una afirmación que aplica para la población general, también hay una alta prevalencia en adolescentes y jóvenes (Parker et al., 2011), que son quienes tienen mayores probabilidades de participar o ser víctimas de crímenes violentos como el abuso sexual, el asalto agravado y los robos en estado de embriaguez.

Los adolescentes que consumen grandes cantidades de alcohol, comparados con los que no lo hacen, tienden a exhibir un mayor número de conductas de riesgo para sí mismos y para los demás (Hingson, Heeren \& Winter, 2006; Miller, Naimi, Brewer \& Jones, 2007); por ejemplo, en un estudio de la Organización Mundial de la Salud (2011), el alcohol resultó ser un factor presente en cerca del $10 \%$ de las muertes entre los jóvenes. Esta cifra se reafirma al observar las tres causas más comunes de mortalidad adolescente, todas asociadas con el consumo de alcohol: accidentes, homicidio y suicidio (Balogun, Koyanagi, Stickley, Gilmour \& Shibuya, 2014). De igual forma, se ha podido demostrar que los adolescentes que presentan consumo abusivo de alcohol están más expuestos a sexo sin protección, lo que conlleva a una mayor probabilidad de embarazo adolescente y de infecciones de transmisión sexual (Stickley, Koyanagi, Koposov, Razvodovsky \& Ruchkin, 2013).

\section{Consumo de alcohol en menores de edad en Colombia}

El último estudio nacional de consumo de sustancias psicoactivas en escolares (Ministerio de Justicia y el Derecho, et al. 2011) indica que el $63 \%$ de los estudiantes de $6^{\circ}$ a $11^{\circ}$ de colegios públicos y privados en Colombia ha consumido alcohol alguna vez en su vida, es decir, que dos de cada tres escolares del estudio lo han probado en algún momento. Específicamente, el $57 \%$ lo hizo en el último año y el 40 $\%$, en el último mes. Esta última prevalencia aumenta con la edad y con el grado escolar, ya que los estudiantes de los últimos grados escolares consumen más en comparación con los de grados inferiores. Asimismo, en dicho estudio no se observaron diferencias con respecto al sexo, pero sí con respecto a las regiones: Caldas, Bogotá, Risaralda, Antioquia y Boyacá ocuparon los primeros lugares, mientras que las ciudades de la Costa Atlántica ocuparon los últimos lugares en consumo de alcohol por parte de adolescentes.

Los resultados del estudio de 2015 (Pérez-Gómez et al., 2015), en lo que se refiere a las prevalencias de vida, año y mes, son muy parecidos a los datos oficiales: $70 \%$, $56 \%$ y $39 \%$, respectivamente; además, se encontró una

1 Existe un estudio más reciente, realizado en 2016, pero a la fecha (mayo de 2018) los datos no han sido publicados. prevalencia, en la última semana, de $19 \%$; la edad de inicio de consumo fue alrededor de los 12 años; la sustancia más frecuentemente asociada al inicio de consumo fue la cerveza; el $31 \%$ se ha embriagado, mientras el $41 \%$ toma máximo dos tragos, el $20 \%$ máximo cuatro, y el $38 \%$ cinco o más tragos; el $65 \%$ considera que es fácil o muy fácil conseguir alcohol, generalmente en tiendas de barrio; el $46 \%$ bebe en presencia de los padres; el $62 \%$ ha estado en fiestas en las que se le sirve alcohol a menores de edad; el $30 \%$ piensa que "fiesta sin alcohol no es fiesta"; el 11\% ha bebido durante varios días seguidos; el $7 \%$ ha tomado antes de llegar al colegio; y el 11\% lo ha hecho dentro del colegio. Aun cuando, en general, estas cifras son menores que las observadas en el estudio de 2008, siguen siendo muy altas y ubican a Colombia como el país con el mayor consumo de alcohol en América Latina.

El presente estudio tuvo como objetivo identificar perfiles que caractericen a los jóvenes consumidores de alcohol a través de un análisis de correspondencias múltiples (ACM), con el fin de contribuir a definir el foco de los programas preventivos.

\section{MÉTODO}

\section{Tipo de investigación}

Estudio transversal descriptivo, con un análisis de correspondencias múltiples (ACM) para manejo de datos. Los métodos exploratorios multivariados, como es el caso de las correspondencias múltiples, permiten representar las relaciones entre grandes conjuntos de variables y grandes grupos de individuos, de manera simultánea. Se denominan exploratorios porque no buscan modelar las relaciones entre las variables ni establecer un valor de probabilidad para la aparición o el cambio en la condición de una variable dependiente. También son llamados métodos de análisis factorial, y entre ellos se encuentran el análisis de componentes principales, el análisis de correspondencias simples y el análisis de correspondencias múltiples, y todos tienen como característica común la extracción rápida de información de un conjunto de datos que por otros medios sería demasiado dispendiosa.

Lo que aquí se plantea es la utilización complementaria de los métodos exploratorios multivariados con el propósito de ilustrar acerca de su potencia como instrumentos para establecer subgrupos (segmentos) dentro de los grupos estudiados, y mostrar distribuciones de variables que pueden ser claves para el mejor entendimiento de los problemas relacionados con el consumo de alcohol y para su respectiva prevención e intervención. 


\section{Participantes}

En el presente artículo se analizaron los datos de 7625 estudiantes que manifestaron haber consumido alcohol por lo menos una vez en la vida, de los 9348 estudiantes de la muestra total del segundo estudio sobre Consumo de alcohol en menores de 18 años en Colombia: siete capitales y dos municipios pequeños (Pérez-Gómez et al., 2015).

En la muestra total, los participantes se encontraban en un rango de edad entre los 10 y 17 años de edad ( $M=14$ años); el tamaño de la muestra fue similar en todos los municipios y osciló entre 942 y 1166 participantes, distribuidos así: Barranquilla (11\%), Bogotá, D.C. (12\%), Bucaramanga (11\%), Cali (11\%), Florencia (10\%), Medellín (12\%) y Tunja (10\%) y dos áreas urbanas de los municipios de Puerto Boyacá (10\%) y Sabanalarga (11\%). En la muestra total, el $53 \%$ de los encuestados fueron mujeres y el $47 \%$, hombres, el $72 \%$ pertenecía a instituciones públicas y el $28 \%$ a instituciones privadas.

Las principales características del diseño muestral fueron: (a) Probabilístico: todos los estudiantes tuvieron una probabilidad diferente de cero de ser seleccionados en la muestra; (b) multietápico: en primer lugar, se seleccionaron las Instituciones Educativas (UPM, $p$ ), en la segunda etapa, grados (UPM, $s$ ), y en la tercera, los alumnos (USM, $t$ ); y (c) conglomerados: las instituciones y los cursos cumplen con las características de un conglomerado y con las características de identificable y cuantificable en campo. Para más información sobre el diseño de la muestra, véase Pérez-Gómez et al. (2015).

\section{Instrumento}

Se utilizó el cuestionario adaptado del aplicado en el primer estudio realizado en el año 2008 por Pérezy Scoppetta (2009). Específicamente, este instrumento fue actualizado para el año 2015, donde se realizaron cambios en cuatro preguntas y se agruparon algunas opciones de respuesta que se encontraron redundantes o con baja tasa de respuesta en el primer estudio con el fin de simplificar el cuestionario. Las cuatro preguntas que fueron reformuladas se describen en la Tabla 1.

El cuestionario está compuesto por 24 preguntas que recolectan información sobre datos sociodemográficos (edad, sexo, grado, tipo de colegio y jornada), patrones de consumo de alcohol y edad de inicio, patrones familiares que facilitan o dificultan el consumo de alcohol, disponibilidad y acceso a bebidas alcohólicas, motivos de consumo, comportamientos de riesgo asociados al uso de alcohol y creencias y actitudes hacia el consumo de alcohol en menores; e incluye opciones de respuesta, en su mayoría de escala nominal, por ejemplo: "Señala qué tipo de licor bebiste la primera vez que tomaste bebidas alcohólicas: Vino, Cerveza, Ron/Aguardiente, Licores importados, Cócteles, Chirrinchi, Chicha, Otros" o "Nunca he tomado bebidas alcohólicas"; también se utilizaron opciones de respuesta tipo Likert, por ejemplo: "El trago te gusta: Mucho, Poco, Nada"; y solo dos preguntas en escala cuantitativa: edad de inicio de consumo de alcohol y cantidad de unidades de cerveza que ingirió la última vez que tomó esta bebida -que posteriormente fueron categorizadas para poderlas incluir en el ACM-. Todas las preguntas fueron tratadas en escala cualitativa. Para más información sobre el cuestionario, véase Pérez-Gómez et al. (2015).

\section{Procedimiento}

El estudio se inició con el contacto a las instituciones educativas y la obtención del consentimiento informado pasivo. Después de que las instituciones educativas informaran a los padres, los estudiantes fueron invitados a participar y dieron su asentimiento. A todos los estudiantes se les informó que la participación sería voluntaria y que se mantendría confidencialidad en todo momento. Después de seleccionar los participantes, se aplicaron los cuestionarios, de manera anónima, en los salones de clase.

Tabla 1

Preguntas modificadas del cuestionario 2008 al cuestionario 2015

\begin{tabular}{|c|c|c|}
\hline \multirow{2}{*}{ Pregunta } & \multicolumn{2}{|c|}{ Estudio } \\
\hline & 2008 & 2015 \\
\hline 1 & $\begin{array}{l}\text { Has tomado bebidas alcohólicas (cerveza, vino, } \\
\text { ron, aguardiente, whisky u otros). }\end{array}$ & $\begin{array}{l}\text { ¿Cuándo fue la última vez que tomaste bebidas al- } \\
\text { cohólicas (cerveza, vino, ron, aguardiente, whisky } \\
\text { u otros)? }\end{array}$ \\
\hline 3 & $\begin{array}{c}\text { Si no tomas bebidas alcohólicas indica los tres } \\
\text { motivos principales. }\end{array}$ & $\begin{array}{l}\text { Si no tomas bebidas alcohólicas indica la razón } \\
\text { principal. }\end{array}$ \\
\hline 16 & $\begin{array}{c}\text { ¿Cuáles son los lugares en los que puedes conseguir } \\
\text { bebidas alcohólicas? (marca los tres más frecuen- } \\
\text { tes). }\end{array}$ & $\begin{array}{l}\text { ¿Cuál es el lugar en el que puedes conseguir bebi- } \\
\text { das alcohólicas más frecuentemente? }\end{array}$ \\
\hline 25 & $\begin{array}{l}\text { Los principales motivos por los que consumes bebi- } \\
\text { das alcohólicas son: (Marca los } 3 \text { más importantes). }\end{array}$ & $\begin{array}{l}\text { El principal motivo por el que consumes bebidas } \\
\text { alcohólicas es: (marca solo el más importante). }\end{array}$ \\
\hline
\end{tabular}

Nota. Tabla adaptada de Pérez-Gómez et al. (2015) pp. 198-200. 


\section{Consideraciones éticas}

Se tuvieron en cuenta todos los principios éticos para investigación con menores de edad establecidos por la ley colombiana; se garantizó la voluntariedad y confidencialidad; y se obtuvo consentimiento pasivo y asentimiento de todos los participantes.

\section{RESULTADOS}

El estudio reveló que el 70 \% de la muestra total había consumido alcohol al menos una vez en la vida (72\% hombres, $67 \%$ mujeres), el $55.7 \%$ en el último año (56.7\% hombres, $54.8 \%$ mujeres), el $38.8 \%$ en el último mes (39.5\% hombres, $38.3 \%$ mujeres) y el $18.9 \%$ en la última semana (19.5\% hombres, $18.3 \%$ mujeres). Al respecto, se observaron diferencias significativas entre hombres y mujeres para la prevalencia de vida $\left(\chi_{(1)}^{2}=25.82, p<0.001\right)$ $\mathrm{y}$ año $\left(\chi_{(1)}^{2}=4.50, p=.034\right)$, pero no para el último mes ni en la última semana.

Como se observa en la Tabla 2, las prevalencias de consumo son muy similares en todas las ciudades, excepto en Sabanalarga, en donde las prevalencias son menores. En los hombres hay una tendencia a consumir más bebidas alcohólicas que las mujeres, diferencias más evidentes en algunas ciudades, como en Barranquilla y Cali.

\section{Análisis de correspondencias múltiples (ACM)}

Como se mencionó en el método, el ACM se realizó en una muestra de 7625 estudiantes que manifestaron haber consumido alcohol por lo menos una vez en la vida de los

Tabla 2

Prevalencias de consumo por ciudades y sexo

\begin{tabular}{|c|c|c|c|c|}
\hline Municipio & Prevalencias & Hombres & Mujeres & Total \\
\hline \multirow{3}{*}{ Barranquilla } & Alguna vez en la vida & 74.1 & 58.4 & 68.0 \\
\hline & En el último año & 60.6 & 43.3 & 50.4 \\
\hline & En el último mes & 36.7 & 26.9 & 30.9 \\
\hline \multirow{3}{*}{ Bogotá } & Alguna vez en la vida & 70.2 & 69.0 & 69.6 \\
\hline & En el último año & 51.5 & 56.7 & 54.1 \\
\hline & En el último mes & 34.6 & 37.7 & 36.1 \\
\hline \multirow{3}{*}{ Bucaramanga } & Alguna vez en la vida & 71.7 & 74.7 & 73.2 \\
\hline & En el último año & 59.3 & 62.9 & 61.2 \\
\hline & En el último mes & 42.6 & 44.2 & 43.4 \\
\hline \multirow{3}{*}{ Cali } & Alguna vez en la vida & 80.3 & 73.6 & 76.7 \\
\hline & En el último año & 66.7 & 62.6 & 64.6 \\
\hline & En el último mes & 46.1 & 43.8 & 44.9 \\
\hline \multirow{3}{*}{ Florencia } & Alguna vez en la vida & 66.3 & 68.1 & 68.0 \\
\hline & En el último año & 47.4 & 54.1 & 51.4 \\
\hline & En el último mes & 34.0 & 37.0 & 35.4 \\
\hline \multirow{3}{*}{ Medellín } & Alguna vez en la vida & 77.9 & 75.4 & 76.7 \\
\hline & En el último año & 63.8 & 63.8 & 63.8 \\
\hline & En el último mes & 49.5 & 52.1 & 50.7 \\
\hline \multirow{3}{*}{ Tunja } & Alguna vez en la vida & 76.8 & 71.6 & 74.4 \\
\hline & En el último año & 62.2 & 61.3 & 61.8 \\
\hline & En el último mes & 45.8 & 44.1 & 45.1 \\
\hline \multirow{3}{*}{ Puerto Boyacá } & Alguna vez en la vida & 73.7 & 71.3 & 72.4 \\
\hline & En el último año & 57.1 & 53.7 & 55.3 \\
\hline & En el último mes & 38.6 & 39.6 & 38.9 \\
\hline \multirow{3}{*}{ Sabanalarga } & Alguna vez en la vida & 57.6 & 43.9 & 50.4 \\
\hline & En el último año & 41.4 & 34.4 & 37.7 \\
\hline & En el último mes & 25.8 & 21.2 & 23.4 \\
\hline
\end{tabular}

Nota. Las prevalencias de último mes y última semana en las entidades territoriales deben leerse con precaución, puesto que están sujetas a eventos que afectan la probabilidad de consumo reciente y a errores de muestreo altos. Tabla adaptada de: Pérez-Gómez et al., (2015) pp. 31-32. 
9348 que respondieron a todas las preguntas de la encuesta. El análisis fue ponderado por los factores de expansión de cada una de las ciudades. Inicialmente, se seleccionaron como variables ilustrativas la edad, el grado, la ciudad, el sexo y tipo de colegio; y, como variables activas, las preguntas relacionadas con el consumo de alcohol:

- Inicio del consumo de alcohol: última vez que consumió alcohol, edad de primer consumo y bebida de inicio.

- Consumo actual: frecuencia de uso de alcohol, gusto por el alcohol, tipo de bebida que toma actualmente, cantidad de alcohol que consume, en dónde y con quién bebe, y motivos de consumo.

- Factores de riesgo y de protección: número de amigos que toman bebidas alcohólicas, ofrecimiento de bebidas alcohólicas a menores de edad en fiestas, consumo de bebidas en fiestas en la casa, consumo en presencia de los padres, facilidad para conseguir bebidas, le han negado la venta de bebidas alcohólicas, actividades preferidas en lugar de beber, regulación de consumo a un amigo.

- Comportamientos problemáticos: ha comprado bebidas alcohólicas, consumo antes de ir al colegio, consumo en el colegio, consecuencias y percepciones respecto al consumo de alcohol.

Las variables ilustrativas y activas seleccionadas corresponden a las mismas utilizadas previamente por Pérez y Scoppetta (2008). Todas las variables fueron analizadas en escala cualitativa y fueron introducidas en un ACM, donde se aplicó el criterio de Benzecri para determinar el número de ejes a retener. En este caso, los factores se obtuvieron al retener el mayor porcentaje de inercia y eliminando las relaciones espurias.

Como resultado, el ACM determinó tres factores (véase Figura 1): (a) el primer y principal factor, que separa a los estudiantes por niveles de consumo - desde estudiantes que probaron alcohol alguna vez en la vida, pero que actualmente no consumen, hasta estudiantes de mayor consumo que manifestaron que el alcohol les gusta mucho y que se han embriagado-; el segundo, relacionado con el inicio del consumo, así como con comportamientos de consumo y de riesgo -donde se separa a quienes tienen un consumo regulado de los que manifestaron haber estado "enguayabados" e iniciaron el consumo hace más de un año con ron o aguardiente-; y (c) el tercero, que separa los casos por tipos de consumo: el consumo en casa-quienes beben vino y que no han estado en fiestas con alcohol- y aquellos que afirmaron que les gusta mucho el trago - quienes tuvieron un inicio de consumo de alcohol a una temprana edad, tienen amigos consumidores, iniciaron con ron o aguardiente y perciben que conseguir alcohol es más o menos fácil-.

Después de establecer los factores, se realizó un análisis a través del método de Ward como forma de agrupamiento de los individuos en grupos homogéneos. El dendograma sugirió que existen dos grupos de consumidores de alcohol: un grupo de bajo consumo, que corresponde al $52 \%$ de los datos incluidos, $y$, el grupo de consumo frecuente, que corresponde al $48 \%$ de la muestra (véase Figura 2). Adicionalmente, se identificaron algunos subgrupos en cada uno de ellos.

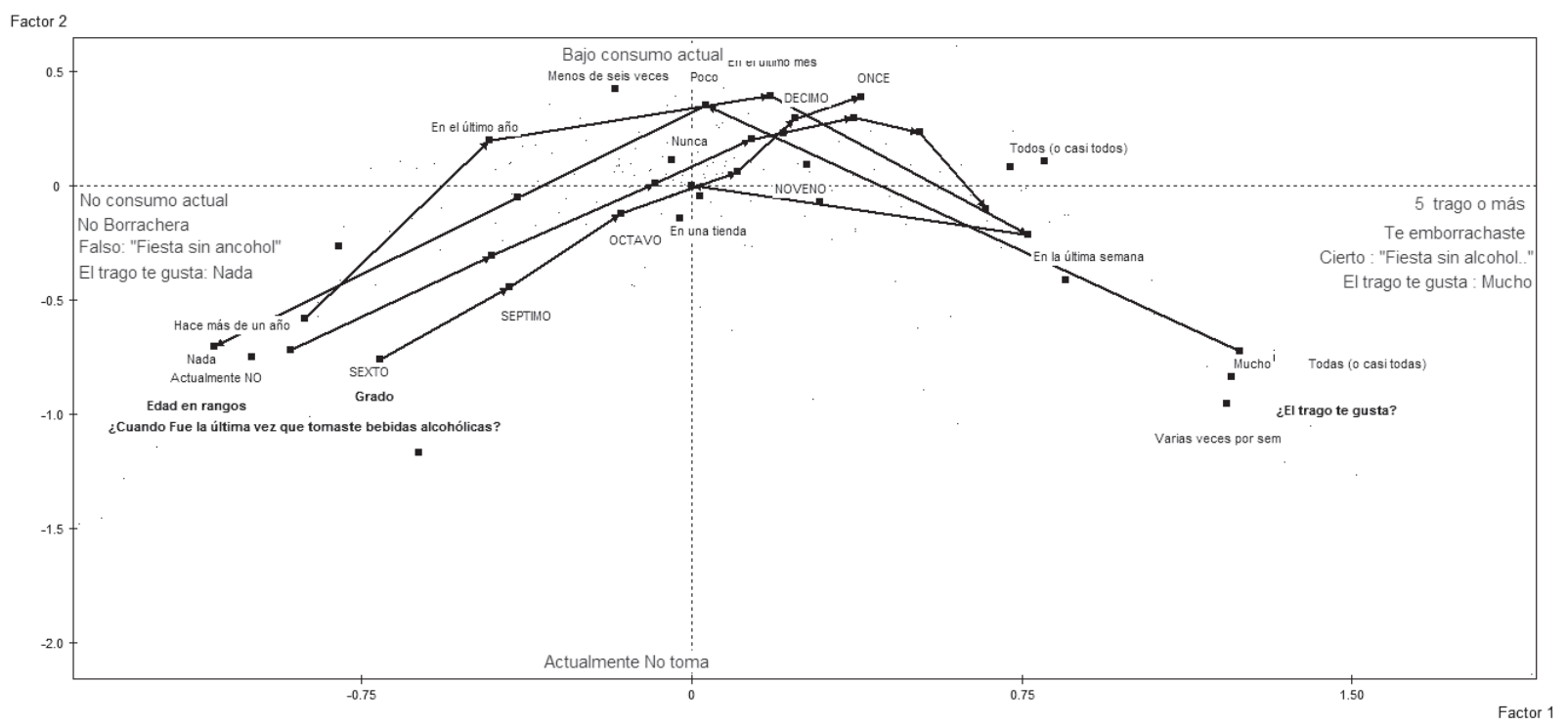

Figura 1. Factor principal de consumo de alcohol por grado escolar. Figura tomada de: Pérez-Gómez et al., (2015) p. 53. 


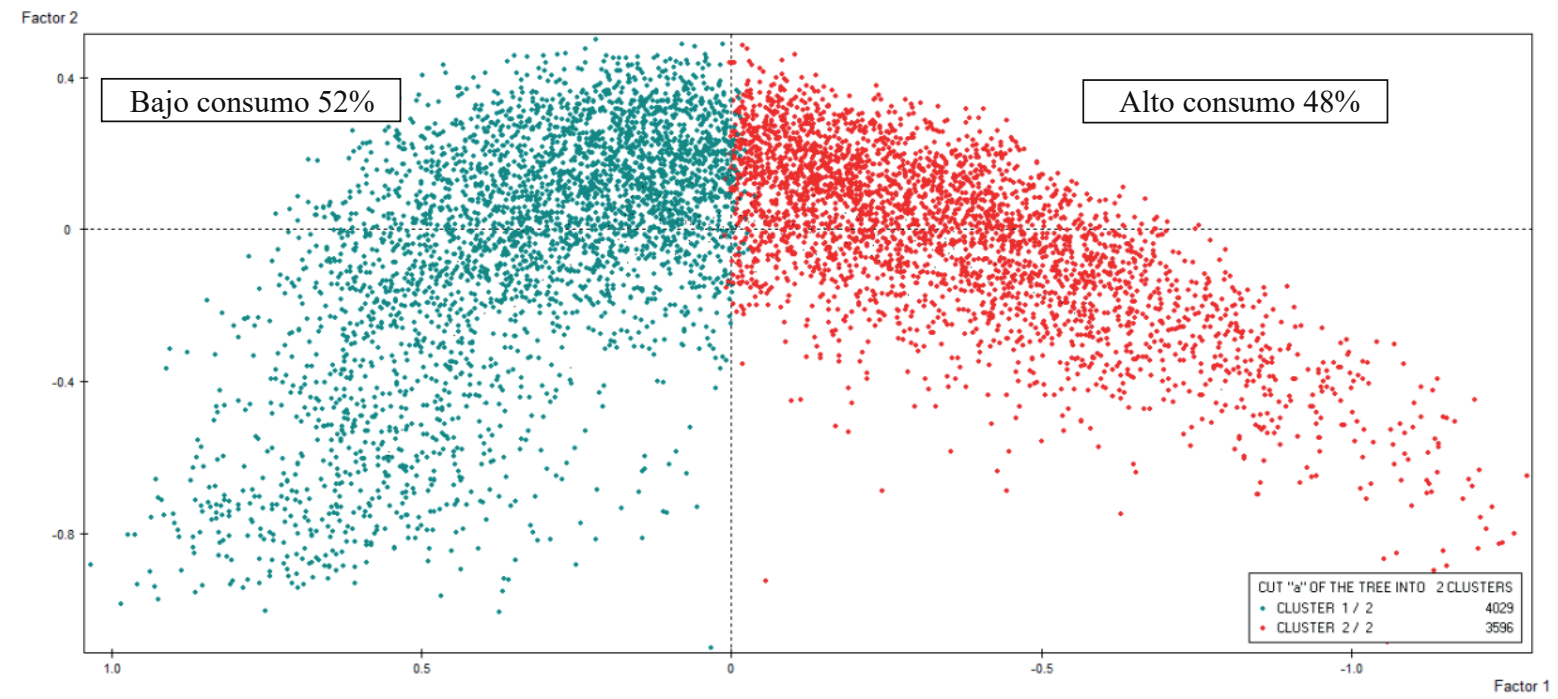

Figura 2. Distribución de los grupos en el mapa factorial. Figura tomada de: Pérez-Gómez et al., (2015) p. 54.

Como se puede observar, en la Figura 2, el análisis indica que en el grupo de bajo consumo, el $80 \%$ no se ha embriagado, el $58 \%$ toma máximo dos tragos, el $45 \%$ ha estado en fiestas sin licor, el $37 \%$ actualmente no consume alcohol, y el $80 \%$ no está de acuerdo con la afirmación "fiesta sin alcohol no es fiesta"; mientras que en el grupo de consumo frecuente hay jóvenes que consumen altas cantidades de alcohol, que han experimentado comportamientos de riesgo, y cerca del $70 \%$ consume cinco tragos o más, el $65 \%$ se ha embriagado, el $45 \%$ consume en las fiestas de sus casas, el $93 \%$ ha estado en fiestas donde sirven bebidas alcohólicas a menores de edad, y más de la mitad $(61 \%)$ está de acuerdo con la afirmación "fiesta sin alcohol no es fiesta".

Posteriormente, se exploró una alternativa de segmentación que mostró cuatro subgrupos: (a) consumo selectivo conformado (29\%, 2039 jóvenes), (b) consumo regular (43\%, 3400 jóvenes), (c) alto consumo (21\%, 1591 jóvenes) y (d) consumo experimental (7 \%, 595 jóvenes); los cuales fueron ordenados por frecuencia y hábitos de consumo (véase Figura 3).

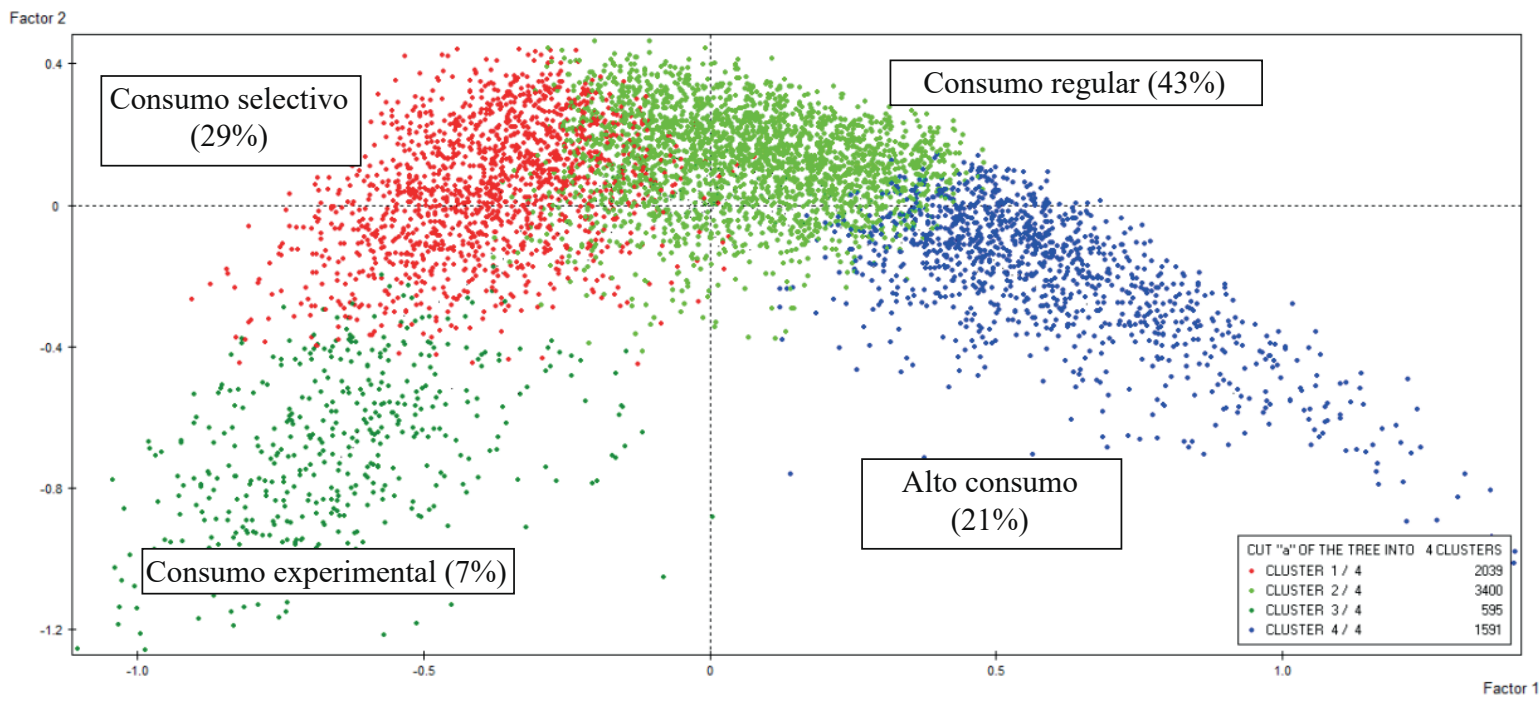

Figura 3. Propuesta segmentación del consumo. Figura tomada de: Pérez-Gómez et al., (2015) p. 54. 
Finalmente, los hallazgos de esta segunda segmentación identifican cuatro grupos, es decir, cuatro categorías que describen los perfiles de consumo de alcohol de los menores encuestados:

- Consumo experimental: personas que no consumen actualmente, pero han consumido alcohol por lo menos una vez en su vida. En este caso, el $57 \%$ fueron mujeres y el $43 \%$, hombres, y no se encontró un patrón según grado escolar, aunque los jóvenes de grado sexto y noveno fueron quienes obtuvieron mayor participación, seguidos por los de octavo y undécimo grado.

- Consumo selectivo: personas que usan alcohol ocasionalmente. Su consumo se limita a celebraciones especiales, el $44 \%$ fueron mujeres y el $56 \%$ hombres, y en su mayoría eran estudiantes de grado sexto, séptimo y octavo.

- Consumo regular: personas con consumo frecuente, alrededor de una vez al mes, y que tienden a embriagarse. En este, el $49 \%$ fueron mujeres y el $51 \%$ hombres, y con respecto a la distribución por grados, la mayoría se encontraba en grados noveno, décimo y undécimo.

- Alto consumo: personas que presentan un consumo en altas cantidades de manera semanal o varias veces al mes, que manifiestan gusto por el alcohol y que se embriagan con frecuencia. En este grupo, el $52 \%$ fueron mujeres y el $48 \%$ hombres, y la mayoría de los jóvenes se encuentra en los grados noveno, décimo y undécimo.

Distribución según el comportamiento de consumo y acceso a bebidas alcohólicas.

En cuanto al comportamiento de consumo según el grupo de consumidores, se encontró que tanto el $54 \%$ de las personas en el grupo de consumo regular como el $92 \%$ de las de alto consumo manifestaron haberse embriagado; mientras que, en los grupos de bajo consumo, la mayoría no lo había hecho. Es de resaltar que el $24 \%$ de los jóvenes del grupo de alto consumo se embriagó por primera vez a los 11 años o menos (véase Figura 4).

En cuanto al acceso a bebidas alcohólicas, se encontró que el $97 \%$ de los jóvenes del grupo alto consumo y el $84 \%$ del consumo regular han estado en fiestas en donde se les sirve alcohol a menores de edad (fenómeno que sucedió en el $55 \%$ del grupo de consumo experimental y el $42 \%$ de consumo selectivo). Consecuente con este hallazgo, se encontró que una proporción importante de los consumidores activos ha bebido alcohol en las fiestas que realizan en los hogares, en comparación con quienes actualmente no consumen (véase Figura 5).

Por otro lado, se encontró que la mayoría de los consumidores activos han bebido alcohol en presencia de los padres: el $87 \%$ de las personas en el grupo de alto consumo, $75 \%$ en el de consumo regular y $65 \%$ en el grupo de consumo selectivo; mientras que quienes no toman bebidas alcohólicas actualmente lo han hecho (beber en presencia de sus padres) en una menor proporción (14\%).

Por último, cerca de la mitad de los jóvenes de todos los grupos le ha pedido a algún adulto que le compre alcohol. Aun cuando esto ocurre frecuentemente en todos los grupos, es más común en el grupo de alto consumo $(84 \%)$ y en el de consumo regular $(67 \%)$.

\section{DISCUSIÓN}

La comparación de los estudios realizados por la Corporación Nuevos Rumbos en 2008 (Pérez-Gómez \& Scoppetta, 2009) y en 2015 (Pérez-Gómez et al., 2015), en

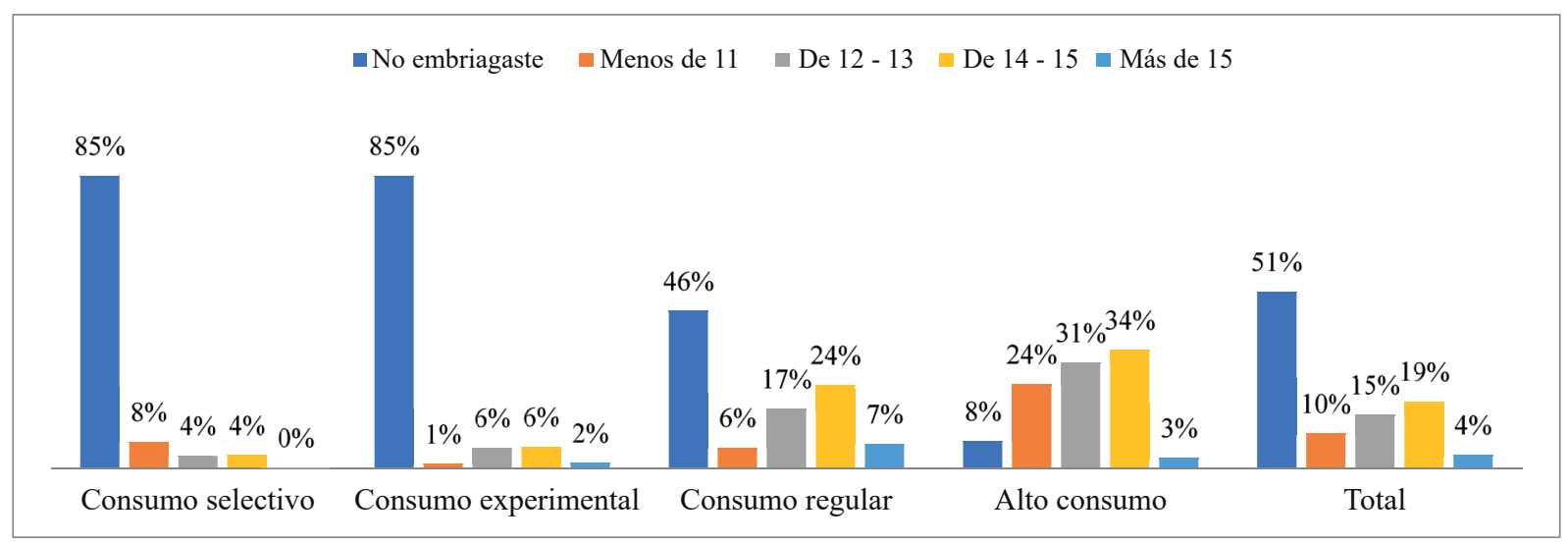

Figura 4. Edad a la que se embriagó por primera vez. Figura tomada de: Pérez-Gómez et al., (2015) p. 56. 


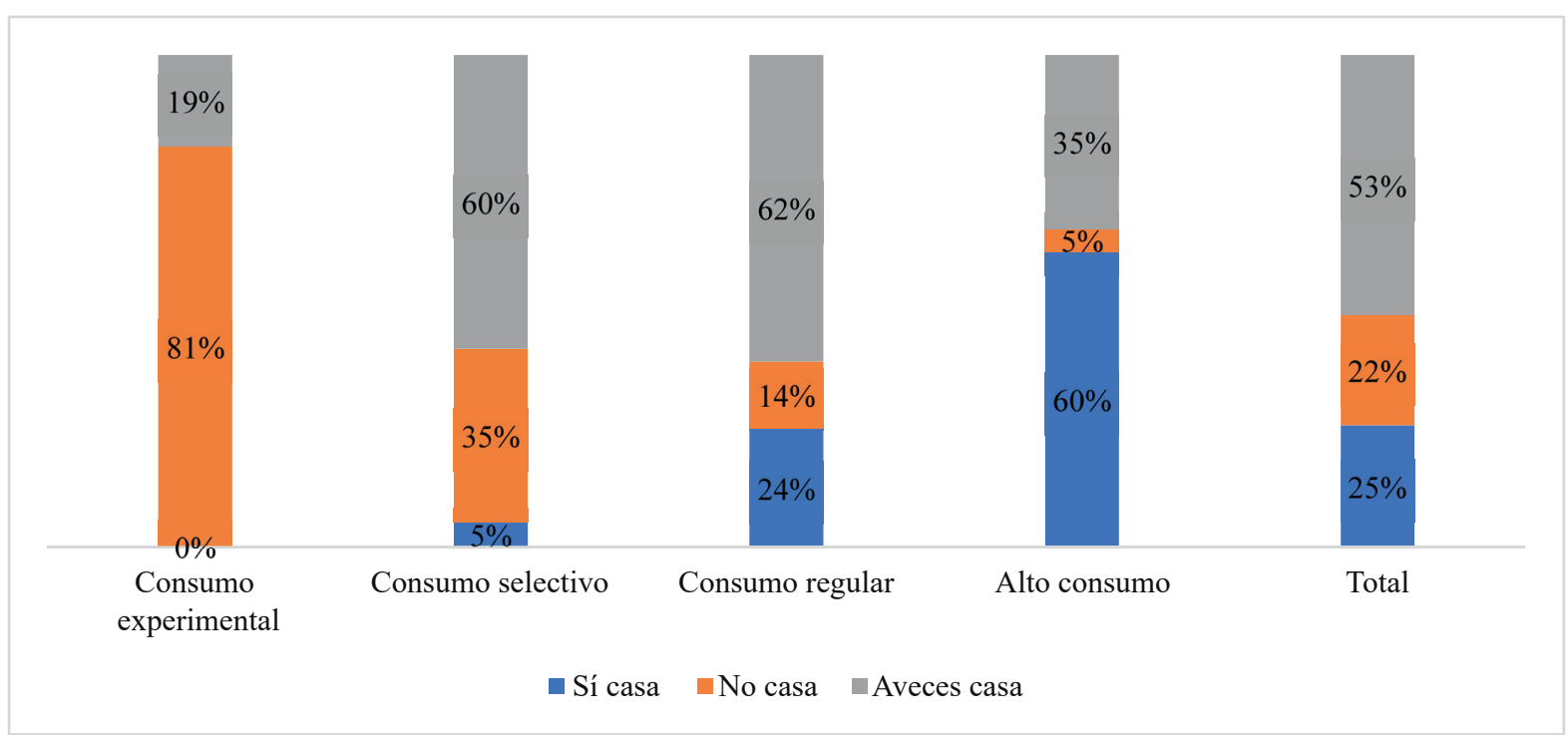

Figura 5. ¿Ha tomado bebidas alcohólicas en las fiestas que realizan en su casa? Figura tomada de: Pérez-Gómez et al., (2015) p. 57.

siete ciudades capitales y dos municipios pequeños, mostró que, aun cuando hubo una tendencia a la disminución, el consumo de alcohol en menores de edad sigue siendo el más alto de América Latina (CICAD/OEA, 2015), y por ello debe ser motivo de atención por parte de las autoridades encargadas de los programas de salud pública, de prevención y de protección de los menores de edad.

En efecto, tal como mostraron Pérez-Gómez et al. (2011), quienes comienzan a consumir alcohol tempranamente tienen hasta 10 veces más probabilidades de tener problemas asociados al consumo de alcohol en la adultez, y cuatro veces más probabilidades de tener problemas con otras sustancias, en comparación con aquellos que empezaron después de los 18 años. Los estudios citados también mostraron que hay un grupo importante (cerca del $10 \%$ ) de adolescentes que consume cantidades muy elevadas de alcohol por ocasión, con una frecuencia también muy alta.

Teniendo esto en cuenta, el análisis de correspondencias múltiples demostró ser un excelente instrumento para construir patrones cuando se trabaja con grandes grupos de variables y grandes muestras. Específicamente, con este procedimiento se analizaron tres factores: el primero separa a los estudiantes por niveles de consumo; el segundo analiza la edad de inicio, comportamientos de consumo y de riesgo; y el tercero separa los casos por tipos de consumo: uno de bajo riesgo y otro de alto riesgo. Sobre esta base, se construyeron dos grupos, cada uno con dos subgrupos: alto consumo, dividido en consumo regular y alto consumo; y bajo consumo, dividido en consumo experimental y consumo selectivo. Las dos categorías intermedias presentan los mismos rasgos, pero en proporciones variables.

Las categorías mencionadas permiten proponer perfiles bastante diferentes: mientras que quienes presentan bajo consumo tienen pocos amigos que beben, no consumen alcohol en su casa ni en presencia de sus padres, no se emborrachan, tienden a tomar solo dos tragos y casi nunca han tenido problemas por tomar; los de alto consumo tienen el perfil opuesto: la mayoría de sus amigos bebe, piensan que "fiesta sin trago no es fiesta", se embriagan con frecuencia, toman en su casa y delante de sus padres, generalmente cinco o más tragos, han tenido problemas relacionados con tomar-como riñas, accidentes, peleas con la pareja y conflicto con los padres-y rechazan el que se sancione a los adultos que les proporcionen alcohol a menores. Hallazgos consistentes con lo encontrado por Fry (2011), Hingson et al. (2006) y Pridemore y Grubesic (2012).

Asimismo, en relación con estas afirmaciones, Becoña (1999), Fagan et al. (2012) y Martínez (2006) demostraron que los adolescentes del grupo de alto consumo tienen actitudes favorables hacia el alcohol, baja percepción de riesgo y creencias positivas sobre su consumo, lo cual también coincide con los hallazgos del presente estudio.

Por otra parte, diferentes investigaciones (Fein et al., 2013; Pascual et al. 2014) han demostrado que los adolescentes con elevados niveles de consumo tienen más probabilidades de tener problemas físicos importantes a nivel del sistema nervioso central, lo que a su vez puede tener expresiones psicosociales, como agresividad, dificultades de aprendizaje y conductas oposicionistas. Dichas conductas, 
identificadas también en este estudio, encuentran soporte en los trabajos de Briones y Woods (2013) y Pilatti et al. (2014) sobre el consumo temprano de alcohol.

Uno de los principales valores de este estudio es haber utilizado un mismo instrumento y un mismo sistema de muestreo (probabilístico, multietápico y por conglomerados), con ocho años de intervalo, en poblaciones de características idénticas y en un número muy parecido, lo que da un mayor valor a las conclusiones. El instrumento adaptado (al cual se le hicieron pocos, pero importantes cambios sobre la base de lo observado en 2008) funcionó mucho mejor que su predecesor.

Una de las limitaciones de este estudio se relaciona con el hecho de que algunos aspectos comportamentales resultantes del abuso de alcohol en la adolescencia no fueron explorados, como lo fue el acoso sexual y las conductas sexuales riesgosas (Hingson et al, 2006; Miller et al., 2007; Parker et al, 2011; Stickley et, 2013). Por tanto, sería conveniente incorporar dichas conductas en el instrumento que se utilice en el futuro.

Por último, sería importante ampliar el número de ciudades en las que se lleve a cabo estos estudios, ya que a pesar de que las muestras son representativas de las ciudades y los municipios en donde se recolectaron los datos, no lo son a nivel nacional. Para concluir, los análisis de este estudio contribuyen para la detección de factores de riesgo y de protección, por lo que pueden resultar de gran utilidad en la formulación de políticas públicas y estrategias preventivas a nivel nacional.

\section{REFERENCIAS}

Balogun, O., Koyanagi, A., Stickley, A., Gilmour, S., \& Shibuya, K. (2014). Alcohol consumption and psychological distress in adolescents: a multi-country study. Journal of Adolescent Health, 54(2), 228-234.

Barr, T., Helms, C., Grant, K., \& Messaoudi, I. (2015). Opposing effects of alcohol on the immune system. Progress in Neuro-Psychopharmacology \& Biological Psychiatry. Doi: http://dx.doi.org/10.1016/j.pnpbp.2015.09.001

Becoña, E. (1999). Bases teóricas que sustentan los programas de prevención de drogas. Madrid: Plan Nacional sobre Drogas.

Briones, T. L., \& Woods, J. (2013). Chronic binge-like alcohol consumption in adolescence causes depression-like symptoms possibly mediated by the effects of BDNF on neurogenesis. Neuroscience, 254, 324-34. Doi:10.1016/j.neuroscience.2013.09.031

Bromley, R., \& Nelson, A. (2002). Alcohol-related crime and disorder across urban space and time: evidence from a British city. Geoforum, 33, 239-254.
Brown, S, A., \& Tapert, S.F. (2008). Adolescence and the trajectory of alcohol use: basic to clinical studies. Annals of the New York Academy of Sciences, 1021, 232-244.

CICAD/OEA (2015). Informe sobre uso de drogas en las Américas 2015. Recuperado de http://www.cicad.oas.org/oid/ pubs/UsoDrogasAmericas_SPA_web.pdf

De Bellis, M. D., Narasimhan, A., Thatcher, D. L., Keshavan, M. S., Soloff, P., \& Clark, D. B. (2005). Prefrontal cortex, thalamus, and cerebellar volumes in adolescents and young adults with adolescent-onset alcohol use disorders and comorbid mental disorders. Alcoholism-Clinical and Experimental Research, 29(9), 1590-1600.

Fagan, A., Hanson, K., Briney, J. S., \& Hawkins, J. D. (2012). Sustaining the Utilization and High Quality Implementation of Tested and Effective Prevention Programs Using the Communities That Care Prevention System. Journal of Community Psychology, 49, 365-377.

Fein, G., Greenstein, D., Cardenas, V. A., Cuzen, N. L., Fouche, J.-P., Ferrett, H., \& Stein, D. J. (2013). Cortical and subcortical volumes in adolescents with alcohol dependence but without substance or psychiatric comorbidities. Psychiatry Research: Neuroimaging, 214(1), 1-8.

Fry, M. L. (2011). Seeking the pleasure zone: Understanding young adult's intoxication culture. Australasian Marketing Journal, 19(1), 65-70. doi:10.1016/j.ausmj.2010.11.009

Hingson R.W., Heeren, T., \& Winter, M.R. (2006). Age at drinking onset and alcohol dependence: Age at onset, duration, and severity. Archives of Pediatrics \& Adolescent Medicine, 160, 739-746.

Jacobus, J., \& Tapert, S.F. (2013). Neurotoxic effects of alcohol in adolescents. Annual Review of Clinical Psychology, 9, 703-721.

Kuntsche, E., \& Labhart, F. (2012). Investigating the drinking patterns of young people over the course of the evening at weekends. Drug and Alcohol Dependence, 124(3), 319324. doi:10.1016/j.drugalcdep.2012.02.001

Liang, W., \& Chikritzhs, T. (2014). Alcohol Consumption during Adolescence and Risk of Diabetes in Young Adulthood. BioMed Research International. Doi: http://dx.doi. org/10.1155/2014/79574

Martínez, E., (2006). Hacia una prevención con sentido. Bogotá: Colectivo Aquí y Ahora.

Miller, J. W., Naimi, T. S., Brewer, R. D., \& Jones, S. E. (2007). Binge drinking and associated health risk behaviors among high school students. Pediatrics, 119(1), 76-85.

Ministerio de Justicia y el Derecho, Ministerio de Educación Nacional, Ministerio de Salud y Protección Social, UNODC \& CICAD/OEA (2011). Estudio nacional de consumo de sustancias psicoactivas en poblacion escolar colombia - 2011 1. Bogotá D. C. (Colombia).

Ministerio de Justicia y el Derecho, Ministerio de Salud y Protección Social \& UNODC (2014). Estudio nacional de consumo de sustancias psicoactivas en Colombia 2013. 
Recuperado de https://www.unodc.org/documents/colombia/2014/Julio/Estudio_de_Consumo_UNODC.pdf

Organización Mundial de la Salud (2011). Global status report on alcohol and health. Geneva: World Health Organization.

Parés, A., \& Caballería, J. (2006). Alcohol y aparato digestivo. Adicciones, 18(1), 51-70.

Parker, R. N., Williams, K. R., McCaffree, K. J., Acensio, E. K., Browne, A., Strom, K. J., \& Barrick, K. (2011). Alcohol availability and youth homicide in the 91 largest US cities, 1984-2006. Drug and Alcohol Review, 30(5), 505-514.

Pascual, M., Pla, A., Miñarro, J., \& Guerri, C. (2014). Neuroimmune activation and myelin changes in adolescent rats exposed to high-dose alcohol and associated cognitive dysfunction: A review with reference to human adolescent drinking. Alcohol and Alcoholism, 49(2), 187-192. Doi:10.1093/alcalc/agt164

Pérez-Gómez, A., \& Scoppetta, O. (2009). Consumo de alcohol en menores de 18 años en Colombia: 2008 un estudio con jóvenes escolarizados de 12 a 17 años en 7 capitales de departamento y dos municipios pequeños. Bogotá: Corporación Nuevos Rumbos. Recuperado de http://scholar. google.com/scholar?hl=en\&btnG=Search\&q=intitle:Co nsumo + de + alcohol + en + menores $+d e+18+a n ̃ o s+e n+C o l$ ombia+:+2008+un+estudio+con+jóvenes+escolarizados + de $+12+a+17+$ años + en $+7+$ capitales + de + departamento $+\mathrm{y}+\mathrm{dos}+$ municipios + pequeños $\# 0$

Pérez-Gómez, A., Scoppetta, O., \& Flórez-Alarcón, L. (2011). Age at onset of alcohol consumption and risk of problematic alcohol and psychoactive substance use in adulthood in the general population in Colombia. The Journal of International Drug, Alcohol and Tobacco Research, 1, 19-24.

Pérez-Gómez, A., Mejía-Trujillo, J., Reyes-Rodríguez, M. F., \& Cardozo-Macías, F. (2015). Consumo de alcohol en menores de 18 años en Colombia: 2015. Bogotá, Colombia: Corporación Nuevos Rumbos.

Pérez-Trujillo, M., Reyes, M.F., Cabrera, L., \& Pérez-Gómez, A. (2016). Changing the beat: Fostering prevention of alcoholrelated violence in night-time leisure zones. International Criminal Justice Review, 26,169-186.
Pilatti, A., Caneto, F., Garimaldi, J. A., Vera, B. D. V., Pautassi, R. M., Hope, A., Aguilar-Jiménez, E. (2014). Binge drinking in adolescents: A review of neurophysiological and neuroimaging research. Alcohol and Alcoholism, 49(2), 198206. Doi:10.1093/alcalc/agt172

Pridemore, W. A., \& Grubesic, T. H. (2012). A spatial analysis of the moderating effects of land use on the association between alcohol outlet density and violence in urban areas. Drug and Alcohol Review, 31(4), 385-393.

Schofield, T. P., \& Denson, T. F. (2013). Alcohol outlet business hours and violent crime in New York State. Alcohol and Alcoholism, 48(3), 363-369.

Shield, K. D., Parry, C., \& Rehm, J. (2014). Chronic diseases and conditions related to alcohol use. Alcohol Research Current Reviews, 35(2), 155-171.

Stickley, A., Koyanagi, A., Koposov, R., Razvodovsky, Y., \& Ruchkin, V. (2013). Adolescent binge drinking and risky health behaviours: Findings from northern Russia. Drug and Alcohol Dependence, 133(3), 838-844.

Szabo, G., \& Mandrekar, H. (2009). A recent perspective on alcohol, immunity and host defense. Alcohol: Clinical and Experimental Research, 33(2), 220-232.

Vicente-Herrero, M. T., López González, Á. A., Ramírez-Iñiguez de la Torre, M. V., Capdevila-García, L., TerradillosGarcía, M. J., \& Aguilar-Jiménez, E. (2015). Cardiovascular risk parameters, metabolic syndrome and alcohol consumption by workers. Endocrinología Y Nutrición (English Edition), 62(4), 161-167. Doi:10.1016/j.endoen.2015.02.009

Ward, R. J., Lallemand, F., \& De Witte, P. (2014). Influence of adolescent heavy session drinking on the systemic and brain innate immune system. Alcohol and Alcoholism, 49(2), 193-197. Doi:10.1093/alcalc/agu002

White, A. M., \& Swartzwelder, H. S. (2006). Age-related effects of alcohol on memory and memory-related brain functions in adolescents and adults. Recent Developments in Alcoholism, 17, 161-176. 